\title{
Direct Detection of Isolevuglandins in Tissues using a D11 scFv-Alkaline Phosphatase Fusion Protein and Immunofluorescence
}

\author{
Cassandra Warden ${ }^{*}, 1$, Alan J. Simmons ${ }^{\star}, 2$, Lejla Pasic ${ }^{3}$, Ashley Pitzer ${ }^{4,6}$, Sean S. Davies ${ }^{4}$, Justin H. Layer ${ }^{5}$, Raymond L. \\ Mernaugh $^{3}$, Annet Kirabo ${ }^{4,6}$ \\ ${ }^{1}$ Vanderbilt Eye Institute, Vanderbilt University Medical Center ${ }^{2}$ Department of Cell and Developmental Biology, Vanderbilt University ${ }^{3}$ Department \\ of Biochemistry, Vanderbilt University ${ }^{4}$ Division of Clinical Pharmacology, Department of Medicine, Vanderbilt University Medical Center ${ }^{5}$ Division of \\ Hematology and Oncology, Indiana University School of Medicine ${ }^{6}$ Department of Molecular Physiology and Biophysics, Vanderbilt University \\ *These authors contributed equally
}

\section{Corresponding Author}

Annet Kirabo

annet.kirabo@vanderbilt.edu

\section{Citation}

Warden, C., Simmons, A.J., Pasic, L., Pitzer, A., Davies, S.S., Layer, J.H., Mernaugh, R.L., Kirabo, A. Direct Detection of Isolevuglandins in Tissues using a D11 scFv-Alkaline Phosphatase Fusion Protein and Immunofluorescence. J. Vis. Exp. (173), e62603, doi:10.3791/62603 (2021).

\section{Date Published}

July 5, 2021

DOI

$10.3791 / 62603$

URL

jove.com/video/62603

\section{Abstract}

Isolevuglandins (IsoLGs) are highly reactive gamma ketoaldehydes formed from $\mathrm{H} 2$ isoprostanes through lipid peroxidation and crosslink proteins leading to inflammation and various diseases including hypertension. Detection of IsoLG accumulation in tissues is crucial in shedding light on their involvement in the disease processes. However, measurement of IsoLGs in tissues is extremely difficult, and currently available tools, including mass spectrometry analysis, are laborious and extremely expensive. Here we describe a novel method for in situ detection of IsoLGs in tissues using alkaline phosphatase-conjugated D11 ScFv and a recombinant phage-display antibody produced in E. coli by immunofluorescent microscopy. Four controls were used for validating the staining: (1) staining with and without D11, (2) staining with bacterial periplasmic extract with the alkaline phosphatase linker, (3) irrelevant scFV antibody staining, and (4) competitive control with IsoLG prior to the staining. We demonstrate the effectiveness of the alkaline phosphatase-conjugated D11 in both human and mouse tissues with or without hypertension. This method will likely serve as an important tool to study the role of IsoLGs in a wide variety of disease processes.

\section{Introduction}

Isolevuglandins (IsoLGs), also known as isoketals, are isomers of the 4-ketoaldehyde family, which are products of lipid peroxidation, and react with and adduct to primary amines on proteins ${ }^{1,2}$. IsoLGs have been implicated in several diseases, including cardiovascular, Alzheimer's, lung and liver diseases, and many types of cancer $^{3}$. IsoLGs have been most extensively studied in their contribution to cardiovascular disease (CVD), which is a significant health and economic burden globally, including the United States. It is estimated that 92.1 million US adults have at least one 
type of CVD, with 2030 estimated projections reaching to $43.9 \%$ of the US adult population ${ }^{4}$. Lowering blood pressure, cholesterol, and smoking cessation reduces the overall risk and occurrence of CVD events ${ }^{5}$.

High blood pressure or hypertension is a major risk factor for cardiovascular disease and affects approximately half of the US population ${ }^{6}$. Previous studies have found that inflammation is an underlying cause of hypertension and that IsoLGs play a role $^{7}$. Hypertensive stimuli, including angiotensin II, catecholamines, aldosterone, and excess dietary salt, induce IsoLG accumulation in antigen presenting cells including dendritic cells (DCs), which in turn activate T cells to proliferate and produce inflammatory cytokines that contribute to hypertension 8,9 .

Previously, IsoLGs have been measured by immunohistochemistry, mass spectrometry, enzyme-linked immunosorbent assay, and flow cytometry ${ }^{10,11}$. To facilitate the measurement of IsoLGs, a single-chain fragment variable (scfv) recombinant antibody (D11) was developed against IsoLGs ${ }^{12}$. Initially, this D11 antibody contained an 11 amino acid E-tag and required a secondary antibody for immunohistochemistry detection ${ }^{11}$. However, it was difficult to find a reliable secondary antibody against the E-tag after the discontinuation of its production by the manufacturer. Therefore, we have developed a reliable protocol for immunofluorescent staining of IsoLGs using D11 conjugated with alkaline phosphatase (D11-AP), which we have demonstrated in mouse and human tissues with and without hypertension.

\section{Protocol}

Vanderbilt University's Institutional Animal Care and Use Committee approved all procedures described in this manuscript. Mice are housed and cared for in accordance with the Guide for the Care and Use of Laboratory Animals. All subjects gave written informed consent before enrolling in the study as approved by the Institutional Review Board of Vanderbilt University. All procedures were performed according to the Declaration of Helsinki.

\section{Preparation of plasmids encoding D11-alkaline phosphatase fusion protein and negative control vectors}

1. Construct a modified version of the pCANTAB5E plasmid $^{13,14}$ in which the single chain fragment variable (scFv) segment is linked at its $3^{\prime}$ end to a sequence encoding bacterial alkaline phosphatase (AP).

NOTE: Immediately downstream of the scFv sequence's Notl restriction site, the modified plasmid no longer contains the coding sequence of the E-tag and instead encodes the linker sequence GGSGGHMGSGG, followed by the sequence for AP (GenBank accession number AXY87039.1, T16-K464) 15,16. Downstream of AP, the plasmid will encode $8 x H$ is and DYKDDDDK tags. At the 3 ' end of the tags, the coding sequence ends with an Amber stop codon. The modified plasmid is called pCANTAB5-AP.

2. Clone D11 scFv (GenBank accession number AAW28931.1) into pCANTAB5-AP with the Sfil/Notl restriction sites.

3. Clone D20 scFv into pCANTAB5-AP with the Sfil/Notl restriction sites.

NOTE: D20 scFv is a negative control designed to assess the tissue interaction pattern of an irrelevant ScFv. This scFv was originally selected by phage display for its ability to interact with a glycan group known as A2. 
4. Generate an "empty" vector by removing the scFv portion from the plasmid entirely and replacing it with an "AP linker" coding sequence consisting of amino acids GGGGSGRAGSGGGGS.

5. Transform all plasmids into competent TG1 E. coli and grow bacteria overnight at $30{ }^{\circ} \mathrm{C}$, on $0.5 \%$ agar plates containing 2xYT supplemented with $100 \mu \mathrm{g} / \mathrm{mL}$ Ampicillin and $2 \%$ glucose (2xYTAG) medium.

NOTE: Strains with the supE gene (such as TG1 $E$. coli) do not suppress the amber stop codon $100 \%$. Estimates range from $0.8-20 \%$ of the time, so there is still a lot of product that terminates just downstream of BAP, as it is intended for these experiments ${ }^{17}$. TG1 E. coli are mainly used for practical reasons, such as the reduction of time and costs, their compatibility with the pCANTAB protein expression system, and their use in phage display, which is commonly conducted in the lab. Downstream of the amber stop codon following BAP is a sequence for genelll. Genelll fusion proteins need to be expressed rarely for phage display to function as intended because scFv-genelll fusion proteins interfere with that particular genelll protein to re-infect naïve bacteria. Therefore, fusion-free genelll proteins need to exist during virion assembly for the generation of functioning phage i.e., scFv-genelll protein products are usually relatively rare inside the bacterium. D11-AP, D20$\mathrm{AP}$, empty vector, and all constructs used in this article are all affected similarly by the occasional genelll fusion protein expression. There are still observed differences in how these proteins behave.

6. Pick an individual colony and inoculate a fresh $5 \mathrm{~mL}$ of 2xYTAG culture. Allow bacteria to grow overnight at 30 ${ }^{\circ} \mathrm{C}$ and shaking at $150 \mathrm{rpm}$.
7. Pellet the cells by centrifugation at $3,000 \times g$ for 10 min at room temperature. Discard the supernatant and resuspend the pellet in $2 \mathrm{~mL}$ of $85 \%$ 2xYTAG and $15 \%$ glycerol.

8. Maintain glycerol stocks in a $-80^{\circ} \mathrm{C}$ freezer.

\section{Protein expression and generation of periplasmic extract}

NOTE: Generation of the periplasmic extract is a commonly used method for protein expression in phage display, mainly because the formation of disulfide bonds is important in ScFv and antibody generation. The method avoids the need to generate lysates (commonly containing inclusion bodies) and ensures that proteins are properly folded. pCANTAB has a glll signal sequence upstream of the scFv portion of the D11BAP fusion protein. The signal sequence ensures that the protein is shuttled to the periplasmic space of the bacterium, and then the signal sequence is cleaved. The periplasmic space provides an oxidizing environment, which is crucial to the proper formation of disulfide bridges. Osmotic shock is used to derive periplasmic extracts because it disrupts the outer membrane enough to release the periplasmic proteins into the surrounding medium, while keeping the bacterium intact.

1. Inoculate TG1 E. coli glycerol stocks containing the relevant plasmids into a $60 \mathrm{~mL}$ culture of 2xYTAG and culture overnight at $30^{\circ} \mathrm{C}$ with shaking at $150 \mathrm{rpm}$.

2. To induce protein expression, pellet bacterial cultures at $3,000 \times g$ for $10 \mathrm{~min}$, resuspend in $60 \mathrm{~mL}$ of $2 \times Y T A$ medium, and culture overnight at $30{ }^{\circ} \mathrm{C}$ with shaking at $150 \mathrm{rpm}$.

NOTE: The switch from 2xTYAG to 2xYTA medium helps in sufficient induction of protein expression ${ }^{18}$. 
When glucose is present in the bacterial medium, the lac promoter is suppressed because bacteria preferentially consume glucose and ignore lactose, as glucose takes less energy to process. When glucose is removed following the medium switch, bacteria rely on the carbohydrates provided by the $2 \mathrm{xYT}$ medium. Yeast extract (the $\mathrm{Y}$ component in 2xYT) contains carbohydrates, among them lactose, and is, therefore, able to drive protein expression through the lac promoter. IPTG is not necessary with the PCANTAB construct and can result in excessive protein expression, along with inclusion bodies that result in non-functional protein.

3. Generate periplasmic extracts via osmotic shock.

1. Pellet bacterial cultures at $3,000 \times g$ for $10 \mathrm{~min}$.

2. Resuspend in $20 \mathrm{~mL}$ of $1 \times \mathrm{xTES}(0.2 \mathrm{M}$ Tris- $\mathrm{HCl} \mathrm{pH}$ 8.0, 0.5 mM EDTA, 0.5 M sucrose) and incubate for $1 \mathrm{~h}$ on ice.

3. Pellet again at $3,000 \times g$ for $10 \mathrm{~min}$, and resuspend in $15 \mathrm{~mL}$ of $0.05 \mathrm{M}$ Tris, $\mathrm{pH}$ 7.6.

4. Incubate this suspension on ice for $1 \mathrm{~h}$, then clarify by centrifugation at $5,000 \times \mathrm{g}$ for $10 \mathrm{~min}$.

5. Transfer supernatants to a fresh tube and store at -20 ${ }^{\circ} \mathrm{C}$ until needed for either purification or direct use in experiments.

6. Assess the cell lysis by the presence of active D11-BAP in the ELISA. Add 3-5 $\mu \mathrm{L}$ of the periplasmic extract to one $\mathrm{mL}$ of pNPP, then observe if a color change happens over the next 10 min (color goes from clear-yellow to intense yellow).

NOTE: The color can be compared side-by-side to a tube of pNPP which has not received any lysate or a tube of pNPP which received lysate of naïve TG1 bacteria. Quantify with absorbance at $405 \mathrm{~nm}$.

\section{Characterization of D11-AP titer in ELISA}

1. Coat a 384-well polystyrene plate overnight at $4{ }^{\circ} \mathrm{C}$ with $25 \mu \mathrm{L} /$ well phosphate-buffered saline (PBS) (1.8 $\mathrm{mM} \mathrm{KH} \mathrm{PO}_{4}, 10 \mathrm{mM} \mathrm{Na} 2 \mathrm{HPO}_{4}, 2.7 \mathrm{mM} \mathrm{KCl}, 137 \mathrm{mM}$ $\mathrm{NaCl}$ ) containing either $5 \mu \mathrm{g} / \mathrm{mL}$ of mouse serum albumin (MSA) (negative control), or $5 \mu \mathrm{g} / \mathrm{mL}$ of IsoLG/MSA (positive control).

2. Empty the plate and tap dry. Wash once with PBS $+0.1 \%$ Tween (PBS-T). Empty the plate and tap dry.

3. Fill the plate with PBS-T as a blocking buffer $(120 \mu \mathrm{L} /$ well). Incubate for $1 \mathrm{~h}$ at room temperature.

4. Empty the plate and tap dry. Apply serial 1:2 dilutions of D11-AP periplasmic extracts at $25 \mu \mathrm{L} /$ well and diluted in PBS-T. Include one well containing only PBS-T as a negative control.

NOTE: Typical dilution ranges for periplasmic extracts are 1:8 - 1:4096. For a $25 \mu \mathrm{L}$ assay volume, start with $50 \mu \mathrm{L}$ of the starting "1:8" concentration: $6.25 \mu \mathrm{L}$ periplasmic extract and $43.75 \mu \mathrm{L}$ PBS-T. Then, perform a 2-fold dilution by removing $25 \mu \mathrm{L}$ of this solution and adding it to the next well containing $25 \mu \mathrm{L}$ of PBS-T and pipette it up and down. This well now contains the "1:16" dilution. Keep repeating the 2 -fold dilution to generate the described 1:2 dilution series.

5. Incubate for $1.5 \mathrm{~h}$ at room temperature.

6. Empty the plate and tap dry. Wash 5 times with PBS-T. Empty and tap dry.

7. Prepare pNPP solution by dissolving $1 \mathrm{~g}$ of pNPP in 1 $\mathrm{L}$ of $930 \mathrm{mM}$ diethanolamine $(98 \%$ stock solution diluted 1:10 in $\mathrm{H}_{2} \mathrm{O}$ ), with $0.5 \mathrm{mM} \mathrm{MgCl}_{2}$ and adjusted to $\mathrm{pH} 9.5$ with $\mathrm{HCl}$. 
8. Apply $25 \mu \mathrm{L} /$ well pNPP solution to develop AP. Incubate for $1 \mathrm{~h}$ at room temperature and determine the absorbance at $405 \mathrm{~nm}$ within each well using a compatible plate reader.

9. Compare the signal generated from the IsoLG/MSA wells to the noise generated from the MSA wells and find the range of dilutions in which the signal is at least 5-fold higher than the noise.

10. Plot this D11-AP signal dilution series on a graph, determine the linear range of the curve, and establish the dilution where $50 \%$ of the signal can be observed.

NOTE: If this dilution is equivalent to approximately 1:1,000, then the D11-AP solution may be used at a concentration of 1:10 in IHC/IF.

\section{Immunofluorescence}

1. Cut serial sections of mouse and human paraffinembedded tissues (5 $\mu \mathrm{m}$ thick) using a microtome and place in a warm water bath $\left(37^{\circ} \mathrm{C}\right)$. Mount tissue sections on glass slides and allow to dry overnight.

NOTE: For this study, aortae were obtained from mice. Colon sections of normotensive and hypertensive humans were obtained from the Vanderbilt Cooperative Human Tissue Network.

2. Immerse slides in xylene three times for $5 \mathrm{~min}$ to deparaffinize tissues.

3. Rehydrate tissues in 2 washes each of $95 \%, 70 \%$ and $50 \%$ ethanol in $\mathrm{H}_{2} \mathrm{O}$.

4. Wash slides in Tris-buffered saline (TBS) with $0.1 \%$ Tween20 (TBST) three times with quick washes by filling the slide holder with TBST then discarding TBST.

NOTE: Hydrated slides can be stored in TBST at $4{ }^{\circ} \mathrm{C}$ for no longer than a week before antigen retrieval.
5. To perform heat-induced antigen retrieval of slides, place slides in pre-heated $\left(80-95{ }^{\circ} \mathrm{C}\right)$ sodium citrate buffer (10 mM sodium citrate, $0.05 \%$ Tween $20, \mathrm{pH} 6.0$ ) and incubate in a pressure cooker set to $4 \mathrm{~min}$ on high pressure for a total antigen retrieval time of $20 \mathrm{~min}$.

6. Remove slides from the pressure cooker and allow them to cool for $20 \mathrm{~min}$ to room temperature.

7. Wash slides in TBST three times with quick washes. NOTE: Slides can be stored in TBST after antigen retrieval for no longer than a week before staining.

8. Add $2 \%$ BSA dissolved in TBST to block slides. Cover slides with a strip of paraffin film and incubate at room temperature for $15 \mathrm{~min}$.

9. Discard the blocking buffer from the slides.

10. Add $200 \mu \mathrm{L}$ of 1:10 D11-AP in TBST to the slides and cover with a strip of paraffin film.

11. Incubate in a humidified chamber to minimize the antibody solution evaporation for $3 \mathrm{~h}$ at room temperature.

12. Wash slides three times in TBST.

13. Develop with a colorimetric or fluorescent alkaline phosphatase developer for immunohistochemistry or immunofluorescence, respectively. Wash slides once with TBST to remove excess developer and prevent further color development.

14. Counterstain slides with Hoechst nuclear stain at $1 \mu \mathrm{g} /$ $\mathrm{mL}$ in PBS for immunofluorescence. Wash slides once in TBST to remove any excess counterstain.

15. Apply coverslips using the mounting medium. 
16. View slides under an inverted light microscope for immunohistochemistry or a confocal fluorescent microscope for immunofluorescence.

\section{Negative controls}

NOTE: Four negative control experiments can be performed to confirm the specificity of D11-AP staining for IsoLG. Negative control experiments should be performed in the same staining set under the same conditions.

1. In the first negative control experiment, incubate tissues with D11-AP diluted in TBST or TBST alone.

2. Incubate tissues with diluted D11-AP in TBST and bacterial periplasmic extract without D11-AP (AP Linker) diluted in TBST.

3. Perform a competitive assay with IsoLG/MSA or nonadducted MSA as previously described ${ }^{12}$.

1. Prepare IsoLG and IsoLG adducted to mouse serum albumin (MSA) as previously described ${ }^{19}$, at a molar ratio of 8 IsoLG: 1 MSA (8:1 IsoLG/MSA).

\section{Dilute D11-AP 1:10 in TBST.}

3. Incubate diluted D11-AP with $50 \mu \mathrm{g} / \mathrm{mL}$ IsoLG/MSA or non-adducted MSA for $1 \mathrm{~h}$ at room temperature.

4. Add D11-AP with IsoLG/MSA or D11-AP with nonadducted MSA to tissues for staining.

4. Use irrelevant ScFv antibody, D20, to stain tissues for the final negative control set.

\section{Representative Results}

In representative experiments, D11 scfv with an alkaline phosphatase conjugation (D11-AP) was used in immunofluorescence to detect IsoLGs present in angiotensin
II-treated mice compared to normal sham mice and humans with hypertension compared to normotensive humans. Mice were treated with angiotensin II at a dose of $490 \mathrm{ng} / \mathrm{kg} /$ min for two weeks, and hypertension was confirmed with increased systolic blood pressure compared to sham mice ${ }^{10}$. To ensure specificity of D11-AP, tissues were stained with or without the presence of D11-AP. As demonstrated by D11AP staining, the aorta of mice with angiotensin II-induced hypertension showed elevated concentration of IsoLGs when compared to control mice (Figure 1). Background staining or autofluorescence was limited, as shown by negative controls that were not stained with D11-AP.

D11-AP was used to detect IsoLGs present in intestinal tissues of human patients with hypertension (HTN) or normotensive humans (NTN). Hypertension status was established from the hospital records as systolic blood pressure above 140 and diastolic blood pressure above $80 \mathrm{mmHg}$. Researchers developing immunofluorescence protocol for D11-AP were blinded to the hypertension status of human tissues. Sections were stained in the presence and absence of D11-AP to ensure antibody specificity and show background staining or autofluorescence. As shown in Figure 2, we found that tissues from patients with HTN had elevated concentrations of IsoLGs compared to patients with NTN. Staining without D11-AP also showed minimal background staining and autofluorescence. Endogenous alkaline phosphatase is expressed by intestinal epithelium, so the limited fluorescence of tissues stained without D11-AP shows the antigen retrieval used in this protocol was sufficient in inactivating endogenous alkaline phosphatase present in tissues. In combination with the results in mice, these results also show that the immunofluorescence protocol effectively 
shows elevated IsoLGs in hypertension when compared to normotensive status.

D11-AP was isolated and stored in the bacterial periplasmic extract. Mouse and human tissues were stained with D11AP and periplasmic extract containing the AP linker without D11 to ensure that other factors that may be present in the periplasmic extract, such as excess or non-conjugated alkaline phosphatase, do not contribute to the staining observed in tissues treated with D11-AP (Figure 3). Tissues stained with D11-AP resulted in brighter staining compared to tissues stained with periplasmic extract. These results confirm that D11-AP is staining the tissues, and the staining is not due to non-conjugated bacterial alkaline phosphatase that can potentially be present in the periplasmic extract and result in false staining of IsoLGs or contribute to background staining.

A competitive control was performed by pre-incubating D11AP with IsoLG adducted to MSA or non-adducted MSA before staining tissues to show the specificity of D11-AP to IsoLG. If D11-AP is specific for IsoLG, the antibody would bind to IsoLG-MSA, resulting in a depleted availability of D11-AP to stain tissues, and D11-AP incubated with non-adducted MSA would have similar staining to normal D11-AP. In tissues stained with D11-AP pre-incubated with the IsoLG competitor, we found diminished staining compared to tissues that were stained with D11-AP without any preincubation (Figure 4). In tissues stained with D11-AP preincubated with non-adducted MSA, we found staining to be similar to staining observed in tissues with D11-AP. These results show the specificity of D11-AP to IsoLGs due to reduced staining of tissues when D11-AP was pre-incubated with IsoLG/MSA, but not nonadducted MSA. In the final negative control, mouse tissue was stained with D11-AP or irrelevant scFv antibody, D20. Staining mouse aorta with D11-AP resulted in strong staining compared to D20 indicating the specificity of D11-AP to IsoLGs in hypertensive aortae (Figure 5). 

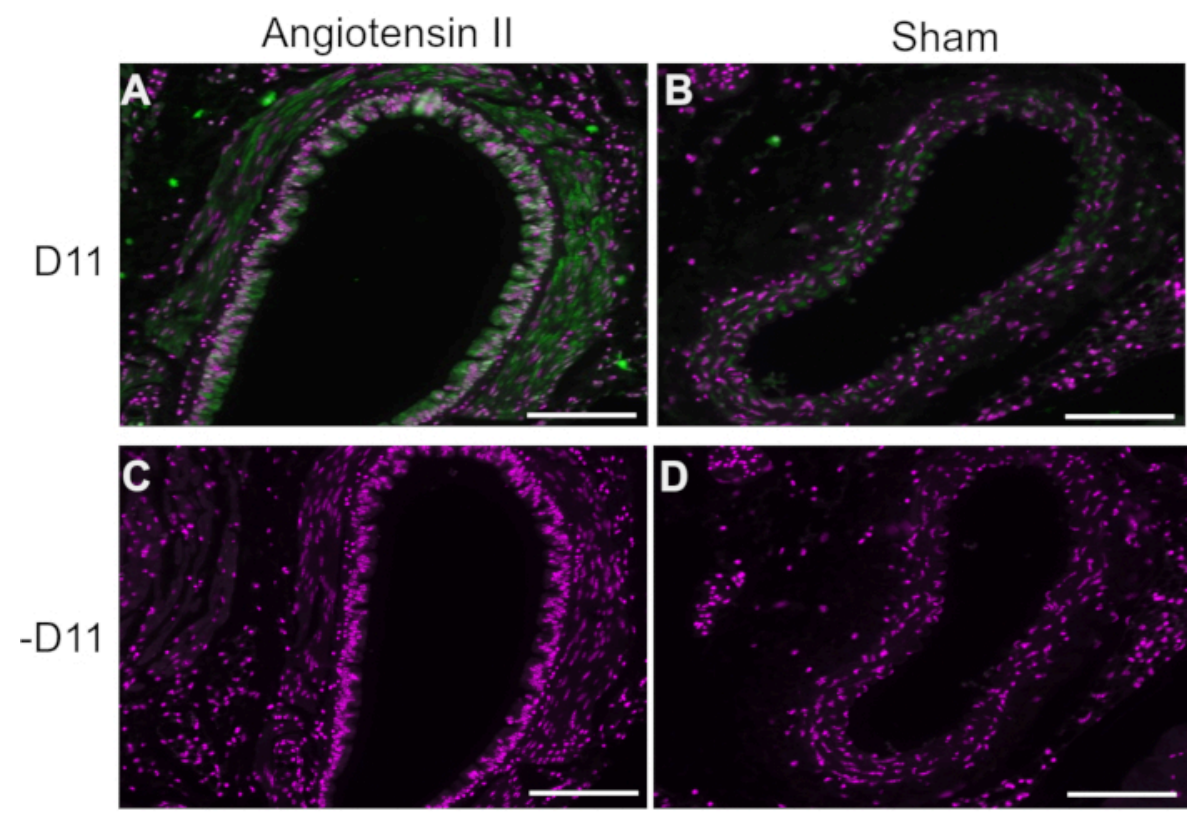

Figure 1: Immunofluorescence of the aorta in hypertensive and normotensive mice. Arteries from angiotensin II and sham infused mice were stained with and without D11-AP (D11) to show presence of IsoLGs. (A) Artery from an angiotensin II treated mouse probed with D11-AP (green) and nuclear counterstain (magenta), (B) Artery from a control sham treated mouse probed with D11-AP, (C) Artery from an angiotensin II treated mouse without D11-AP, (D) Artery from a control sham treated mouse without D11-AP (scale bar $=100 \mu \mathrm{m}$ ). Please click here to view a larger version of this figure. 

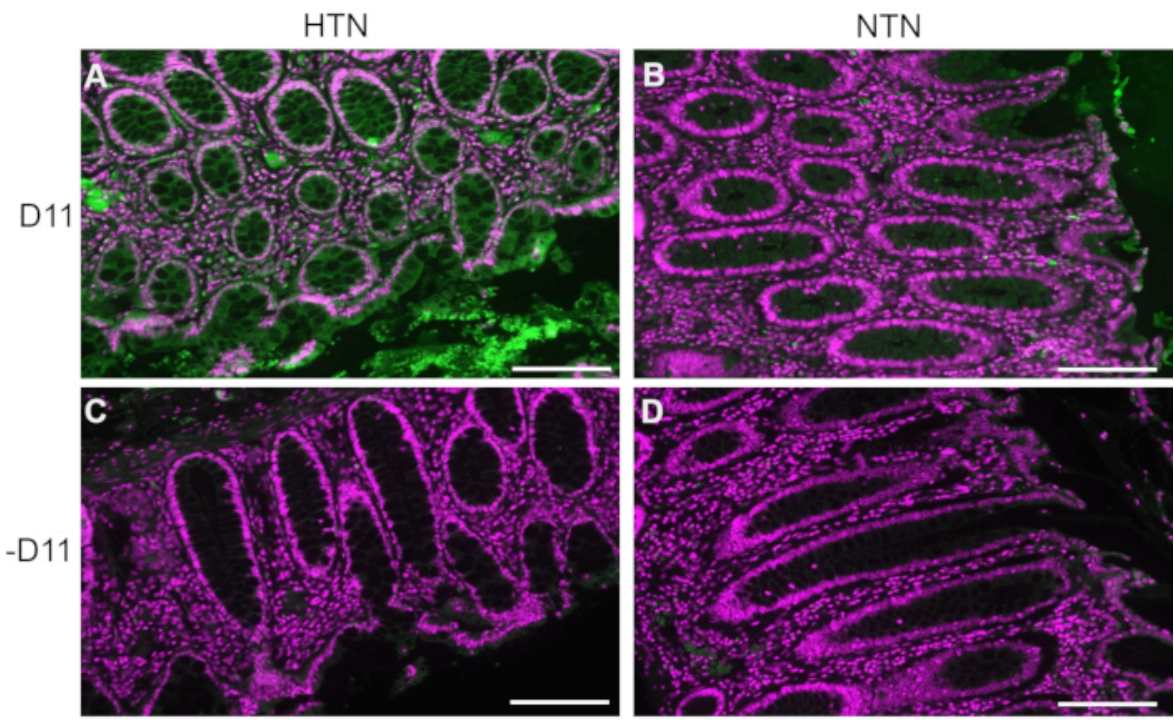

Figure 2: Immunofluorescence of human intestinal tissues from hypertensive and normotensive patients. Tissues from patients with hypertension (HTN) and normotensive humans (NTN) were stained with and without D11-AP (D11) to show presence of IsoLGs in patients with HTN. (A) HTN tissues stained with D11-AP (green) and nuclear counterstain (magenta), (B) NTN tissues stained with D11-AP, (C) HTN tissues stained without D11-AP, (D) NTN tissues stained without D11-AP (scale bar $=100 \mu \mathrm{m}$ ). Please click here to view a larger version of this figure. 


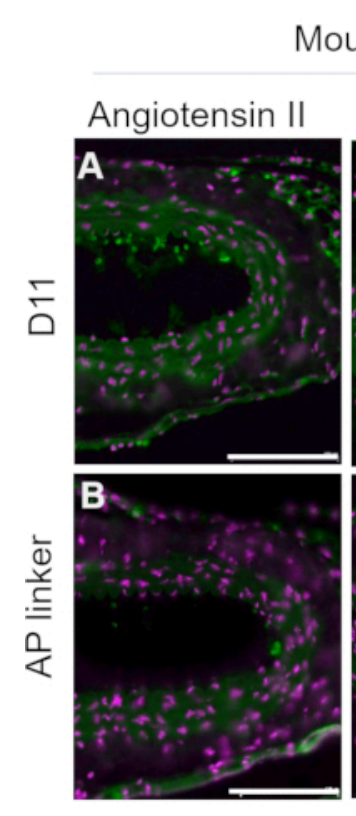

Mouse
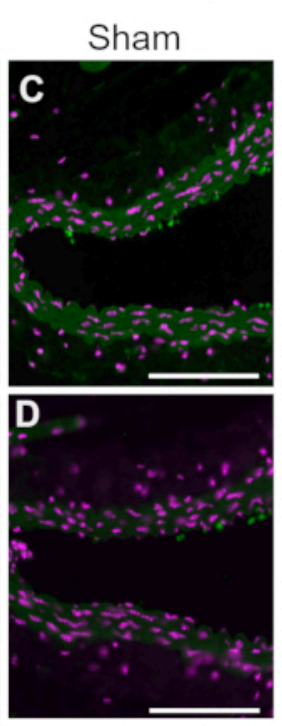

Human

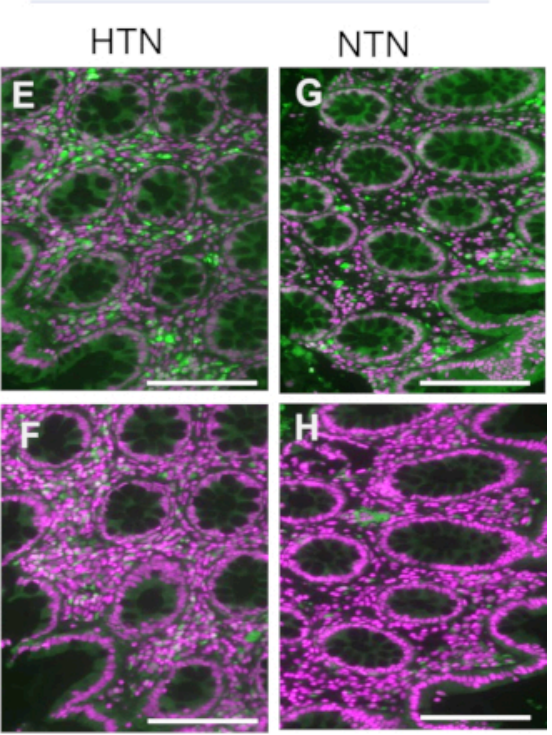

Figure 3: Mouse and human tissues stained with periplasmic extract with and without D11-AP. Mouse and human tissues were stained with periplasmic extracts with and without D11-AP. Images show limited staining of tissues with periplasmic extract without D11-AP, which show staining is mostly due to D11-AP binding rather than another component that may be present in periplasmic extract. (A) Ang mouse aorta stained with D11-AP (green) and nuclear counterstain (magenta), (B) Ang mouse aorta stained with periplasmic extract, (C) Sham mouse aorta stained with D11-AP, (D) Sham mouse aorta stained with periplasmic extract, (E) hypertensive human intestinal tissue stained with D11-AP, (F) hypertensive human intestinal tissue stained with periplasmic extract, $(\mathbf{G})$ normotensive human intestinal tissue stained with D11-AP, (H) normotensive human intestinal tissue stained with periplasmic extract (scale bar $=100 \mu \mathrm{m}$ ). Please click here to view a larger version of this figure. 

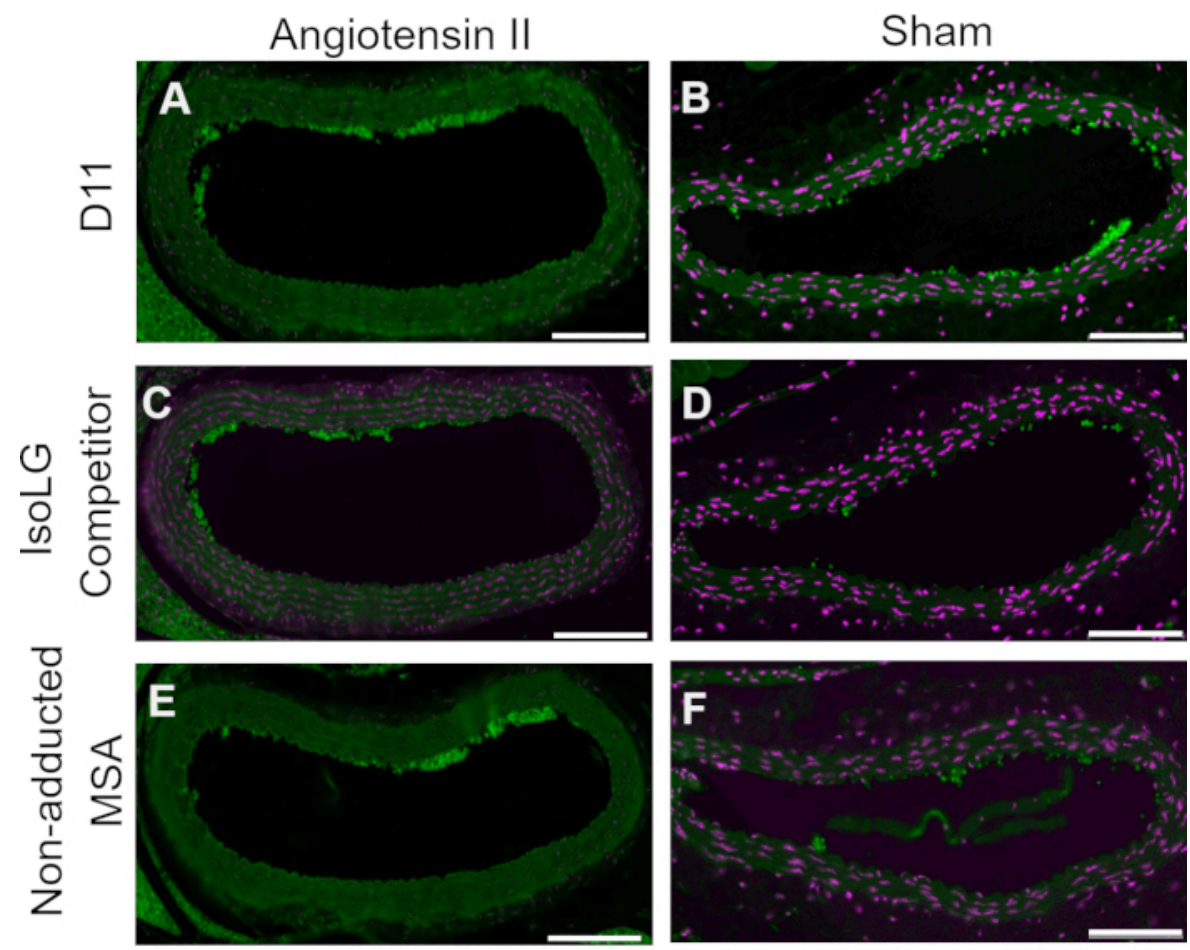

Figure 4: Competitive control in vessels of Ang and Sham mice. In this competitive control, D11-AP was pre-incubated with IsoLG-adducted MSA or non-adducted MSA. D11-AP without any pre-incubation was used as a control. These results show the specificity of D11-AP to IsoLGs because there is reduced staining of tissues with the IsoLG-MSA competitor compared to D11-AP. This reduction is due to IsoLG and not MSA because the non-adducted MSA pre-incubation resulted in staining similar to D11-AP control. Angiotensin II (A) and Sham (B) mouse aortae stained with D11-AP (green) and nuclear counterstain (magenta), Angiotensin II (C) and Sham (D) mouse aortae stained with D11-AP after incubating with IsoLGMSA, Angiotensin II (E) and Sham (F) mouse aortae stained with D11-AP after incubating with non-adducted MSA (scale bar $=100 \mu \mathrm{m})$. Please click here to view a larger version of this figure. 

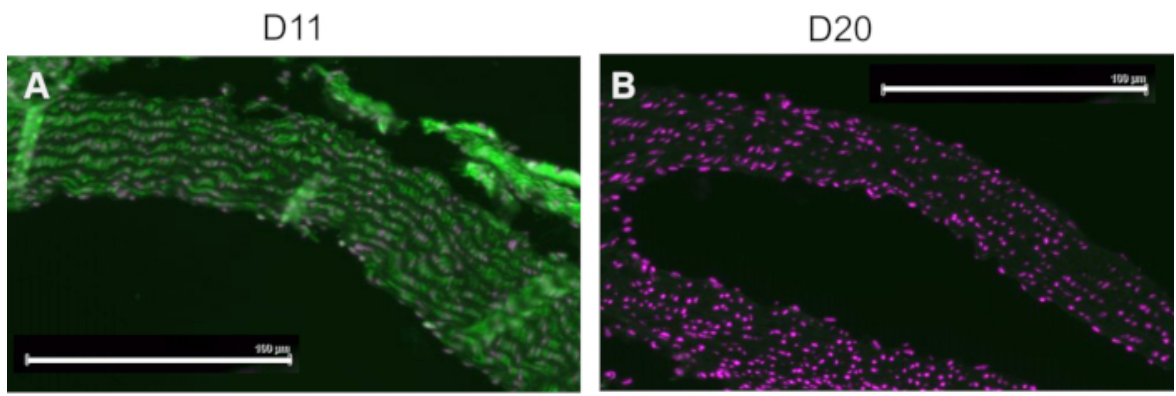

Figure 5: Mouse aortae stained with D11 and irrelevant scFv, D20. Mouse tissue were stained with D11-AP and compared to an irrelevant control antibody, D20, which is specific for glycoprotein A2. Staining of tissue with D11-AP (green) and nuclear counterstain (magenta) (A) resulted in intense immunofluorescence compared to D20 (green) and nuclear counterstain (magenta) (B) which indicates specificity of D11-AP to IsoLGs (scale bar =100 $\mu \mathrm{m}$ ). Please click here to view a larger version of this figure.

\section{Discussion}

D11 has been used extensively to detect IsoLG-adducted proteins in cells or tissues as a marker for inflammation or oxidative stress in disease ${ }^{8,9,20}$. Previously, D11 contained an $\mathrm{E}$ tag and IHC development required the use of a secondary anti-E tag antibody conjugated with $\mathrm{HRP}^{10,20,21}$. Here, we have developed and optimized protocol for detection of IsoLG-adducted proteins using the D11 antibody conjugated with alkaline phosphatase in place of the Etag, which eliminates the need for a secondary antibody incubation.

To determine the specificity of D11-AP, four negative control experiments were performed. We performed the protocol without the presence of D11 and had minimal development. These results have a two-fold indication: endogenous alkaline phosphatase is not contributing to development, and the staining observed is due to D11 and not another contributing factor. Next, we stained slides with the AP linker without D11. This experiment resulted in little staining, which indicates free
AP or other factors in the periplasmic extract is not causing the stain we observe in the presence of D11. To ensure the specificity of D11 to IsoLG, we preincubated D11-AP with purified IsoLG before staining slides. We saw a decrease in development which indicates that the D11-AP was bound to IsoLG protein, thus exhausting the amount of free D11-AP to bind to IsoLG present in the tissue. Finally, to ensure D11AP was binding to IsoLG and not the MSA protein IsoLG was bound to, we preincubated D11-AP with MSA only. There were no changes in development, indicating D11-AP was not binding to MSA but the IsoLG protein. Lastly, researchers developing the staining protocol were blind to hypertensive status of human intestinal tissue. The differences in staining observed between patients with hypertension and patients with normotension were not due to bias and have been previously described 22,23 .

Although our protocol for detection of IsoLG-adducted proteins using the D11 antibody conjugated with alkaline phosphatase in place of the E-tag is rigorous and robust 
and eliminates the need for a secondary antibody incubation, it has some limitations. One limitation is that we used D11 conjugated with alkaline phosphatase in the periplasmic extract, and there could be false staining of endogenous alkaline phosphatase in the periplasmic extract or certain tissues, such as intestine ${ }^{24}$. However, the first step to develop this protocol included disabling endogenous alkaline phosphatase that may be present in tissues ${ }^{25}$. Initially, cold acetic acid, BME, and Levamisole ${ }^{26}$ were tested for efficiency. None of these completely diminished the presence of active endogenous alkaline phosphatase. Heat has been used to deactivate alkaline phosphatase ${ }^{27}$, so we tested heat deactivation of alkaline phosphatase in different buffers. We found heating mounted and hydrated slides in citrate buffer eliminated most endogenous alkaline phosphatase. Slides were developed initially using a Chemiluminescent/ Fluorescent Substrate, but when imaged without this substrate, there was a high amount of autofluorescence. VectorRed is a substrate which develops in the presence of alkaline phosphatase to produce a chromogen which can be visualized in the Texas Red/TRITC channel range. Using this substrate, we were able to more easily observe signal above background autofluorescence. Care should be taken during the staining process to minimize artifactual staining. Drying of tissues on slides after hydration until imaging has resulted in heightened development. D11-AP should be aliquoted and stored at $-20{ }^{\circ} \mathrm{C}$. Multiple freeze-thaw cycles should be avoided when working with D11-AP. Phosphate-buffered saline (PBS) can also affect the enzymatic activity of alkaline phosphatase and should not be used as a wash buffer ${ }^{28}$. As with any antibody-based approach, thorough testing and optimization must be carried out to ensure that staining is specific, and that signal is not over or under amplified.
In conclusion, we have developed a powerful, rigorous, and robust optimized protocol to detect IsoLG-adducted proteins using the D11 antibody conjugated with alkaline phosphatase in place of the E-tag. This protocol presents several advantages: First, using D11 as an alkaline phosphatase fusion protein is cheaper. D11 was originally derived from a phage antibody library that could not be commercialized and was expensive to purify. Although D11 in E. coli periplasmic extract could provide an inexpensive alternative, it was ineffective in most assays. Second, the alkaline phosphatase fusion approach allows D11 scfv to have a useful reporter ${ }^{15}$ (alkaline phosphatase) fused to it and would not need to be purified for use in immunoassays as substrates are commercially available. Third, the E. coli alkaline phosphatase forms dimers ${ }^{29}$. So D11, when fused to the alkaline phosphatase would also form dimers and this increase the antibody's avidity and binding activity ${ }^{30}$. Finally, D11 conjugated with alkaline phosphatase in periplasmic extract can easily be cleaned using Cibacron Blue Sepharose. D11 has a high isoelectric point ( 9.2 pH). As such, it is positively charged and can bind to Cibacron Blue through pi-cation interactions. Most of the impurities in the E. coli periplasmic extract can be eluted off the resin. The D11 conjugated with alkaline phosphatase can then be eluted using high salt $(\sim 1.5 \mathrm{M} \mathrm{NaCl})$ in water. The eluted D11 conjugated with alkaline phosphatase is quite stable at 4-8 ${ }^{\circ} \mathrm{C}$ in the high salt solution. Thus, we have developed a protocol which not only makes the D11 antibody available at a low cost, but also eliminates the extra steps and need for the secondary antibody incubation. This protocol facilitates reproducible measurement of IsoLGs, which accumulate in tissues in multiple diseases where increased oxidative stress plays a role. 


\section{Disclosures}

The authors have nothing to disclose.

\section{Acknowledgments}

This work was supported by National Institutes of Health grants K01HL130497, R01HL147818, R01HL144941, and R03HL155041 to A.K. We thank the Digital Histology Shared Resource - Vanderbilt Health Nashville, TN https://www.vumc.org/dhsr/46298 for visualization and slide scanning.

\section{References}

1. Brame, C. J., Salomon, R. G., Morrow, J. D., Roberts, L. J., 2nd. Identification of extremely reactive gammaketoaldehydes (isolevuglandins) as products of the isoprostane pathway and characterization of their lysyl protein adducts. Journal of Biological Chemistry. 274, 13139-13146 (1999).

2. Brame, C. J., et al. Modification of proteins by isoketalcontaining oxidized phospholipids. Journal of Biological Chemistry. 279, 13447-13451 (2004).

3. May-Zhang, L. S. et al. Scavenging reactive lipids to prevent oxidative injury. Annual Review of Pharmacology and Toxicology. 61, 291-308 (2021).

4. Benjamin, E. J. et al. Heart disease and stroke statistics-2019 update: A report from the American Heart Association. Circulation. 139, e56-e528 (2019).

5. Collins, D. R. et al. Global cardiovascular risk assessment in the primary prevention of cardiovascular disease in adults: Systematic review of systematic reviews. BMJ Open. 7, e013650 (2017).
6. Whelton, P. K. et al. 2017 ACC/AHA/AAPA/ABC/ACPM/ AGS/APhA/ASH/ASPC/NMA/PCNA Guideline for the prevention, detection, evaluation, and management of high blood pressure in adults: Executive summary: A report of the American College of Cardiology/American Heart Association Task Force on clinical practice guidelines. Circulation. 138, e426-e483 (2018).

7. Patrick, D. M., Van Beusecum, J. P., Kirabo, A. The role of inflammation in hypertension: Novel concepts. Current Opinion in Physiology. 19, 92-98 (2021).

8. Davies, S. S. et al. Isolevuglandins as mediators of disease and the development of dicarbonyl scavengers as pharmaceutical interventions. Pharmacology and Therapeutics. 205, 107418 (2020).

9. Dixon, K. B., Davies, S. S., Kirabo, A. Dendritic cells and isolevuglandins in immunity, inflammation, and hypertension. American Journal of Physiology-Heart and Circulation Physiology. 312, H368-H374 (2017).

10. Kirabo, A. et al. DC isoketal-modified proteins activate $\mathrm{T}$ cells and promote hypertension. Journal of Clinical Investigation. 124, 4642-4656 (2014).

11. Yan, H. P, et al. Isolevuglandins as a gauge of lipid peroxidation in human tumors. Free Radical Biology and Medicine. 106, 62-68 (2017).

12. Davies, S. S. et al. Localization of isoketal adducts in vivo using a single-chain antibody. Free Radical Biology and Medicine. 36, 1163-1174 (2004).

13. Shen, Z. et al. Single-chain fragment variable antibody piezoimmunosensors. Analytical Chemistry. 77, 797-805 (2005).

14. Hennig, E. E., Mernaugh, R., Edl, J., Cao, P., Cover, T. L. Heterogeneity among Helicobacter pylori strains 
in expression of the outer membrane protein BabA. Infections and Immunity. 72, 3429-3435 (2004).

15. Martin, C. D. et al. A simple vector system to improve performance and utilisation of recombinant antibodies. BMC Biotechnology. 6, 46 (2006).

16. Han, Z., Karatan, E., Scholle, M.D., McCafferty, J., Kay, B.K. Accelerated screening of phage-display output with alkaline phosphatase fusions. Combinatorial Chemistry and High Throughput Screening. 7, 55-62 (2004).

17. Miller, J. Handbook for a short course in bacterial genetics. Cold Spring Harbor Laboratory. New York. (1992).

18. Nair, R. et al. Yeast extract mediated autoinduction of lacUV5 promoter: An insight. New Biotechnology. 26 (6), 282-288 (2009).

19. Davies, S. S., Amarnath, V., Roberts L. J., 2nd Isoketals: Highly reactive $\mathrm{y}$-ketoaldehydes formed from the $\mathrm{H} 2-$ isoprostane pathway. Chemistry and Physics of Lipids. 128 (1-2), 85-99 (2004).

20. Ngwenyama, $\mathrm{N}$. et al. Isolevuglandin-modified cardiac proteins drive CD4+ T-Cell activation in the heart and promote cardiac dysfunction. Circulation. 143 (12), 1242-1255 (2021).

21. Prinsen, J. K. et al. Highly reactive Isolevuglandins promote atrial fibrillation caused by hypertension. Basic to Translational Science JACC. 5 (6), 602-615 (2020).

22. Ferguson, J. F. et al. High dietary salt-induced dendritic cell activation underlies microbial dysbiosis-associated hypertension. JCI Insight. 5 (13), e126241 (2019).

23. Madhur, M. S. et al. Hypertension: Do inflammation and immunity hold the key to solving this epidemic? Circulation Research. 128 (7), 908-933 (2021).
24. Estaki, M., DeCoffe, D., Gibson, D. L. Interplay between intestinal alkaline phosphatase, diet, gut microbes and immunity. World Journal of Gastroenterology. 20 (42), 15650-15656 (2014).

25. Millán J. L. Mammalian alkaline phosphatases: From biology to applications in medicine and biotechnology. John Wiley and Sons. (2006).

26. Ponder, B. A., Wilkinson, M. M. Inhibition of endogenous tissue alkaline phosphatase with the use of alkaline phosphatase conjugates in immunohistochemistry. Journal of Histochemistry and Cytochemistry. 29 (8), 981-984 (1981).

27. Goldstein, D. J., Rogers, C. E., Harris H. Expression of alkaline phosphatase loci in mammalian tissues. Proceedings of the National Academy of Science U.S. A. 77 (5), 2857-2860 (1980).

28. Ramos-Vara, J. A. Technical aspects of immunohistochemistry. Veterinary Pathology. 42 (4), 405-426 (2005).

29. Coleman, J. E. Structure and mechanism of alkaline phosphatase. Annual Review of Biophysics and Biomolecular Structure. 21, 441-483 (1992).

30. Harper K., Toth, R. L., Mayo M. A., Torrance. L. Properties of a panel of single chain variable fragments against Potato leafroll virus obtained from two phage display libraries. Journal of Virological Methods. 81 (1-2), 159-168 (1999). 